\title{
Appropriate Referral Timing To Specialized Palliative Care Service: Survey of Bereaved Families of Cancer Patients Who Died in Palliative Care Units
}

Keita Tagami ( $\square$ keita.tagami.d7@tohoku.ac.jp)

Tohoku University School of Medicine: Tohoku Daigaku Daigakuin Igakukei Kenkyuka Igakubu https://orcid.org/0000-0001-8081-5979

Kento Masukawa

Tohoku University School of Medicine: Tohoku Daigaku Daigakuin Igakukei Kenkyuka Igakubu

Akira Inoue

Tohoku University School of Medicine: Tohoku Daigaku Daigakuin Igakukei Kenkyuka Igakubu

Tatsuya Morita

Seirei Mikatahara Hospital: Seirei Mikatahara Byoin

Yusuke Hiratsuka

Tohoku University School of Medicine: Tohoku Daigaku Daigakuin Igakukei Kenkyuka Igakubu

Mamiko Sato

Tohoku University Hospital: Tohoku Daigaku Byoin

Katsura Kohata

Tohoku University Hospital: Tohoku Daigaku Byoin

Noriaki Satake

Tohoku University Hospital: Tohoku Daigaku Byoin

Yoshiyuki Kizawa

Kobe University Hospital: Kobe Daigaku Igakubu Fuzoku Byoin

Satoru Tsuneto

Kyoto University Graduate School of Medicine Faculty of Medicine: Kyoto Daigaku Daigakuin Igaku Kenkyuka Igakubu

Yasuo Shima

Tsukuba Medical Center Hospital

Mitsunori Miyashita

Tohoku University School of Medicine: Tohoku Daigaku Daigakuin Igakukei Kenkyuka Igakubu

\section{Research Article}

Keywords: Integration of oncology and palliative care, Specialized palliative care, Referral timing of palliative care, Early palliative care, Cancer patient experience

Posted Date: June 30th, 2021

DOl: https://doi.org/10.21203/rs.3.rs-585422/v1

License: (c) (1) This work is licensed under a Creative Commons Attribution 4.0 International License. Read Full License

Version of Record: A version of this preprint was published at Supportive Care in Cancer on August 21st, 2021. See the published version at https://doi.org/10.1007/s00520-021-06493-2. 


\section{Abstract}

Few studies have investigated appropriate referral timing of specialized palliative care (SPC) from the perspective of cancer patients' and families' experiences. We aimed to clarify appropriate SPC referral timing for patients with advanced cancer and their families. We used data from a nationwide bereaved family survey in Japan. We sent a questionnaire to 999 bereaved families of cancer patients who died in 164 palliative care units (PCUs) and analyzed the first SPC referral timing and how patients evaluated it. We defined SPC as outpatient or inpatient palliative care service comprising certified palliative care physicians, advanced-practice nurses, and multidisciplinary practitioners. Finally, $51.6 \%(n=515)$ of all responses were analyzed. The SPC referral timing was evaluated as appropriate (26.1\%), late or too late (20.2\%), early or too early (1.2\%), and none of these (52.5\%). Of these, $32.3 \%$ reported that they were referred to an SPC when diagnosed with advanced or incurable cancer or during anti-cancer treatment, and $62.6 \%$ reported they were referred after anti-cancer treatment. Patient-perceived appropriateness of SPC referral timing was associated with their good death process. After excluding "none of these" responses, a significantly higher proportion of respondents who reported being referred to SPC at diagnosis and during anti-cancer treatment evaluated the response timing as appropriate, compared to those who reported being referred after anti-cancer treatment. Appropriate timing for SPC referrals relates to quality of death; findings suggest that appropriate timing is at the time of diagnosis or during anti-cancer treatment.

\section{Introduction}

Clinicians need to know appropriate timing for specialized palliative care service (SPC) referral based on patient and family reported outcomes derived from their experiences through cancer diagnosis, anti-cancer treatment, and end of life care; however, few studies have investigated the appropriate SPC referral timing. Some studies surveyed patients visiting outpatient clinics or medical practitioners [1-6]; however, actual referral timing and patients' evaluation of the appropriateness of the timing were not investigated.

There is growing evidence supporting a need to integrate palliative care and oncological care, especially early access to SPC for patients with advanced cancer [7-12]. Those have demonstrated that early SPC involvement is associated with quality of life, symptom burden, depression, mood, patient and caregiver satisfaction, and possibly survival, and that it positively impacts end of life quality care indicators [7-12]. In Japan, the National Cancer Control Act has actively promoted palliative care for cancer patients right from diagnosis to improve destress, symptom burden, and quality of life. However, few studies have examined data on when Japanese patients with advanced cancer are typically referred to SPC.

This study surveyed bereaved families of cancer patients to identify SPC referral timing by analyzing the referral timing experience of cancer patients and their families. Our primary aim was to identify when physicians first referred patients to SPC and how patients evaluated the timing of that referral. Additionally, we investigated differences in patients' evaluation of their SPC referral timing based on when they were referred: 1) at diagnosis or during anti-cancer therapy, or 2) after anti-cancer treatment. We also developed a hypothesis that there may be a relationship between patient-perceived appropriateness of SPC referral timing and their quality of death.

\section{Methods}

\section{Subjects and procedure}

This study was performed as part of a nationwide survey of bereaved family members of cancer patients in Japan, which evaluated quality of care, quality of death of patients, and outcomes of bereaved family members, such as depression and complex grief (the Japan Hospice and Palliative Care Evaluation Study 4: J-HOPE4) [13]. This multicenter study surveyed the family members of patients with cancer who had died in three palliative care settings (14 acute care hospital, 187 inpatient palliative care units (PCUs), and 14 home hospice services). Ethical approval for the study was granted by the institutional review boards of Tohoku University Hospital and all participating institutions (Approval No. 2018-2-290). The inclusion criteria were: 1) the patient died of cancer, 2) the patient was aged 20 years (the age at which one is considered an adult in Japan) or older, and 3 ) the bereaved family member was aged 20 years or older. The exclusion criteria were: 1) the patient received palliative care for fewer than 3 days; 2 ) the bereaved family member was unavailable or could not be identified; 3) death was associated with treatment or occurred in an intensive care unit; 4) the potential participant suffered serious psychological distress, as determined by the primary physician or a nurse; and 5) the potential participant was incapable of completing the self-reported questionnaire because of health issues, such as cognitive impairment or visual disability [13]. The present study surveyed the family members of patients who had died in PCUs.

Between May and June 2018, we mailed a questionnaire to the primary caregivers of the patients who had died more than three months before, as determined from each patient's hospital medical chart according to the study protocol [13]. If individuals did not wish to participate, we requested that they return the questionnaire indicating "no participation." We sent a reminder to nonresponding individuals one month after the initial mailing. The subjects were asked their demographic information (age, sex, and relationship to the patient), interval between patient death and study, and length of patient's cancer struggles (diagnosis, treatment, and end of life). The patients' demographic information was collected from medical records and included age, sex, primary tumor site, and length of stay in the PCU (days). Completing and returning the questionnaire were regarded as consent to participate in the study.

\section{Questionnaires}

We developed an ad-hoc questionnaire based on a literature review $[1,2,4,16-19]$. We assessed the questionnaire's content validity by full agreement of the authors and confirmed the face validity using a pilot test with five family members and five medical practitioners. We defined SPC as an outpatient palliative care clinic or inpatient palliative care team comprising certified palliative care physicians, advanced-practice nurses, and multidisciplinary palliative care staff (psycho-oncology physicians, pharmacists, medical social workers, rehabilitation specialists, dentists, etc.) who support patients (Figure 1) [1, 2, 8, 14, 15]. 
We first identified the actual timing physicians first referred patients to SPC and inquired the bereaved families about it (Figure 1): 1) at diagnosis of advanced or incurable cancer; 2) during anti-cancer treatment; 3 ) no further standard anti-cancer treatment options but independent activities of daily living (ADL); 4) no further standard anti-cancer treatment options and dependent ADL (defined according to a Karnofsky Performance Status Scale score $\leq 40$, where $0=$ dead and 100 = healthy); and 5) no referral [20]. We then asked bereaved families how patients had evaluated their SPC referral timing on a 5-point scale ranging from "too early" to "early," "appropriate," "late," "too late," and "none of these."

Second, we examined the bereaved families' retrospective perceived appropriate SPC referral timing, based on their experience in supporting patients, according to the timing shown in Figure 1; they could be imaged cancer patients with no physical and/or psychological distress and responded either "Agree" or "Disagree." Additionally, we asked the bereaved families to identify, in retrospect, what they or the patient most needed SPC help with, among the following: "physical distress," "sleepless and psychological distress," "family support," "daily life and economic problems," "information about the treatment and assistance with decision making," "end-of-life care," and "none of these."

We evaluated quality of death using the short form of the Good Death Inventory (GDI) [21, 22]. We identified important concepts relating to a good death and included 10 core and 8 optional domains and 54 attributes concerning physical and psychological comfort, living in a favorite place, maintaining hope and pleasure, good relationship with medical staff, not feeling like a burden to others, a good relationship with family, independence, environmental comfort, being respected as an individual, and a feeling of fulfillment at life's completion. Respondents evaluate each attribute using a seven-point Likert scale (1: absolutely disagree, 2: disagree, 3: somewhat disagree, 4: unsure, 5: somewhat agree, 6: agree, and 7: absolutely agree). The total score is calculated by summing the scores for all attributes, with higher total scores indicating a good death. The questionnaire's reliability and validity have been confirmed [21-23].

\section{Statistical analyses}

Descriptive statistics, including proportions (percentages), mean values with standard deviation (SD), and median values with interquartile range (IQR) or range, were used to summarize patient demographics, length of cancer treatment, length of PCU stay, and GDI scores.

The primary outcome was appropriate SPC referral timing based on cancer patients' and their families' evaluations. For analysis, the first SPC referral timing was categorized into two groups: at diagnosis of advanced or incurable cancer and during anti-cancer treatment were combined and coded as "referral at diagnosis or during cancer treatment"; no further standard anti-cancer treatment options but independent ADL and no further standard anti-cancer treatment options and dependent ADL were combined and classified as "referral after cancer treatment." Furthermore, patients' evaluations of their first SPC referral timing was classified into three groups; "late" and "too late" were coded as "evaluated as late," "early" and "too early" were coded as "evaluated as early," and "appropriate" was coded as "evaluated as appropriate." Chi-squared tests were used to test for differences in the proportion of "evaluated as appropriate" between respondents who answered, "referral at diagnosis or during cancer treatment" and "referral after cancer treatment." We excluded "none of these" responses. Chi-squared tests were also used to test for differences in the proportion of "referral at diagnosis or during cancer treatment" between respondents who answered, "evaluated as appropriate" and "evaluated as late."

To investigate the relationship between patient-perceived appropriateness of their SPC referral timing and quality of death, we compared GDI scores between respondents who answered, "evaluated as appropriate" and "evaluated as late" using the Student's T tests. Similarly, we compared these scores between bereaved families who reported "referral at diagnosis or during cancer treatment" and "referral after cancer treatment" using the Student's T tests. Clinically meaningful differences were also assessed by Cohen's $\mathrm{d}$.

The threshold for statistical significance was set at $p<0.05$. All analyses were performed using GraphPad Prism, version 7.0 (GraphPad Software, San Diego, CA, USA).

\section{Results}

Of the 999 questionnaires sent to bereaved family members, 620 were completed and returned (response rate $62.1 \%$ ). Of these, 105 responses were excluded because the individuals declined to participate, or the returned questionnaires had missing data, leaving 515 responses (51.6\%) for analysis. Table 1 presents patients' and their families' characteristics. In patients, mean age (SD) was 76.0 (11.3), and 55.1\% were female. Lung (24.3\%) was the most common primary tumor site, followed by esophagus and stomach (13.2\%), and colon and rectum (12.4\%). One to three years was the most common duration of anti-cancer treatment (31.1\%), followed by more than three years (26.0\%) and less than three months (15.3\%). The median days from last admission to PCU until death were 20 (IQR, 10 - 41). In bereaved family members, mean age (SD) was 62.9 (11.6), 66.8\% were female, children accounted for $41.7 \%$, and spouses accounted for $39.2 \%$. Median days from patient death to completion of the study questionnaire were 323.5 (IQR, 265.5 - 409).

\section{Timing when physicians first referred patients to SPC}

Among the respondents $(n=489)$ who reported physicians' first SPC referral timing, $20.9 \%(n=102)$ responded that referral occurred at diagnosis of advanced or incurable cancer and $11.4 \%(n=56)$ responded that referral occurred during anti-cancer treatment; whereas $36.2 \%$ ( $n=177)$ responded that referral occurred when there were no further standard anti-cancer treatment options but the patient had independent ADL, and $26.4 \%(n=129)$ responded that referral occurred when there were no further standard anti-cancer treatment options and the patient had dependent ADL. An additional 5.1\% reported they had never been referred to SPC during the patients' life. There were no significant differences between patients' demographic characteristics, duration of anti-cancer treatment, or length of PCU stay in participants who responded, "referral at diagnosis or during cancer treatment" or "referral after cancer treatment" (data not shown).

\section{How patients evaluated their first SPC referral timing}

Processing math: $100 \%$ 
Among the respondents $(n=495)$ who reported patients' evaluations of their first SPC referral timing, 26.1\% ( $n=129)$ evaluated the SPC referral timing as appropriate, $11.7 \%(n=58)$ evaluated it as late, and $8.5 \%(n=42)$ evaluated it as too late. Only $0.8 \%(n=4)$ respondents evaluated the SPC referral timing as early and only $0.4 \%(n=2)$ as too early. About half of bereaved families $(n=260,52.5 \%)$ responded "none of these." There were no significant differences in patients' demographic characteristics, duration of anti-cancer treatment, or length of PCU stay in participants who responded, "evaluated as appropriate" and "evaluated as late" (data not shown).

\section{Relationship between actual first SPC referral timing and patients' evaluations}

Figure 2 presents patients' evaluation of their first SPC referral timing, as reported by bereaved family members, categorized according to their actual SPC referral timing. After excluding "none of these," the frequency of "evaluated as appropriate" included $69.3 \%$ of respondents who reported "referral at diagnosis or during cancer treatment" and $48.9 \%$ of those who reported "referral after cancer treatment." There were significantly more "evaluated as appropriate" responses in participants who reported "referral at diagnosis or during cancer treatment" than those who reported "referral after cancer treatment" ( $p<0.01)$. In participants who responded with "evaluated as appropriate" and "evaluated as late," the percentage of "referral at diagnosis or during cancer treatment" was significantly higher in the former than in the latter $(43.3 \%$ and $20.7 \%$, respectively; $p<0.01)$.

\section{Bereaved families perceived appropriate SPC referral timing}

We asked the bereaved families about their perceived appropriateness of SPC referral timing based on their experience supporting patients; the results are presented in Table 2. More than $70 \%$ of these respondents agreed that cancer patients could consult with SPC at any time even in the absence of physical and/or psychological distress.

\section{What bereaved families should have discussed with SPC in retrospect}

When patients' families looked back at their experience, they reported that they should consult with SPC to get help with "physical distress" ( $\mathrm{n}=232,45.0 \%$ ), "end of life care" ( $n=216,41.9 \%)$, "information about the treatment and assistance with decision making" ( $n=191,37.1 \%)$, "sleepless and psychological distress" ( $n=93,18.1 \%)$, "family support" ( $n=88,17.1 \%$ ), and "daily life and economic problems" ( $n=62,12.0 \%)$. Only 12.8\% ( $n=66)$ responded "none."

\section{Relationship between SPC referral timing and quality of death}

Investigating the relationship between patient-perceived appropriateness of their SPC referral timing and their quality of death, those who responded "evaluated as appropriate" had significantly higher GDI scores than those who responded "evaluated as late" (mean (SD), 85.2 (12.6) vs 78.9 (13.1); p< 0.01; Cohen

$s d=0.49)$. GDIsC or ed $\Leftrightarrow$ erencesbetweenparticipantswhoresponded, "referralatdiagnosis or dur $\in$ gcancertreatment" and "referralafte $\mathrm{s} d=0.18)$

\section{Discussions}

To the best of our knowledge, this is the first study to employ a cross-sectional nationwide survey of bereaved families of cancer patients who died in PCUs to investigate the actual timing of physician referral to SPC and how patients with advanced cancer and their families evaluated the referral timing. The strength of our findings is that they reflect cancer patients and their families' experience of cancer diagnosis, anti-cancer therapy, and end of life care. Previous Japanese studies also examined bereaved families' perceived appropriateness of initial PCU referral timing [18]; however, few studies have reported the actual SPC referral timing, including both outpatient palliative care clinics and inpatient palliative care teams, or patients and family members' evaluations regarding the appropriateness of referral timing for patients with advanced cancer.

We found that $32.3 \%$ of respondents reported the actual SPC referral timing as occurring either at advanced or incurable cancer diagnosis or during anticancer treatment. The National Cancer Control Act was implemented in Japan in 2007. Since then, SPC has included providing cancer patients and their families with help following the cancer diagnosis, to improve patients' quality of life. A basic education program, the Palliative Care Emphasis Program on Symptom Management and Assessment for Continuous Medical Education (PEACE program) [15, 28-31] was developed for all physicians engaged in cancer care to help support primary palliative care. Further, a feasibility study of Japanese patients with advanced lung cancer presented evidence that early SPC involvement could improve patients' quality of life, depression, and mood [32]. These results, coupled with our findings, show that SPC clinical activity in Japan has increased over the last 10 years, and cancer patients are finding it easier to access SPC.

We also found SPC involvement at advanced or incurable cancer diagnosis or during anti-cancer treatment was evaluated as more appropriate than "referral after cancer treatment" based on their cancer experiences. Additionally, more than 70\% of patient families who experienced SPC agreed that "referral at diagnosis or during cancer treatment" was appropriate regardless of whether cancer patients were experiencing physical and/or psychological distress. Hui et al. reported that the appropriate SPC referral criteria suggested by experts on palliative care were: within three months of an advanced cancer diagnosis for patients with median survival of one year or less, and progressive disease despite second-line chemotherapy [1]. Recently, Wong et al. reported 54\% of patients visiting their palliative and supportive care clinic judged that the appropriate SPC referral timing was at the time of cancer diagnosis and $24 \%$ judged it to be at the start of the first chemotherapy; whereas only $7 \%$ judged that the appropriate SPC referral timing was when there were no more cancer treatment options [3]. Hoerger et al. reported that more than $50 \%$ of newly diagnosed advanced lung or gastro-intestinal cancer patients and their families emphasized rapport, symptom management, coping, and illness understanding as important factors [25]. Many studies have demonstrated the effectiveness of early SPC involvement and integrating oncology and palliative care, based on patient reported outcomes, such as better quality of life, end of life care delivery, communication, symptom and mood management, preventing depressive symptoms, receiving aggressive end of life care, satisfaction with care, and longer Processing math: $100 \%$ 12, 24-27]. Our findings support the appropriate referral time-based criteria by Hui et al. and importance of earlier referral to SPC. 
Although studies have demonstrated the importance of early SPC involvement and oncology and palliative care integration, SPC referral timing may be still occurring too late, suggesting an important unmet need for cancer patients and their families that could be provided through SPC. We found that about $60 \%$ of those who reported "referral after cancer treatment," and 50.4\% of those who reported "referral after cancer treatment" were "evaluated as late" after excluding the "none of these" responses. Furthermore, Watanabe et al. reported that in a tertiary cancer center in Canada, the median time between SPC consultation and death was two months [33]. Although SPC clinical activities in Japan have become more popular, medical practitioners should continue improving SPC access at any time to make it more convenient for cancer patients and their families even if they are newly diagnosed with advanced or incurable cancer or are receiving anti-cancer treatment.

Some studies reported that the appropriate need-based SPC referral criteria included suffering from severe destress (physical, psychological, and spiritual distress) or a need for end-of life care planning (where disease progression status included no further cancer treatment options, poor prognosis, and dependent ADL) [2, 4-6]. However, we could not examine the appropriate need-based referral criteria, and further efforts are necessary to investigate it based on patients and theirfamilies cancer experience. We also present that patients' perceived appropriateness of SPC referral timing may be associated with a good death process. A recent study demonstrated early SPC may be inadequate [34]; thus, we should seek the time-and need-based suitable SPC referral timing which was perceived as appropriately timed by cancer patients.

This study's strength lies in the nationwide questionnaire data from family members of cancer patients and in its response rate, which was more than $50 \%$. However, this study has some limitations. First, as this research was based on data from bereaved family members, the findings may not be directly attributable to patients. Cancer patients' beliefs and perceptions are most accurate when described by the patients themselves rather than retrospectively described by family members. Additionally, the retrospective design may have resulted in recall bias. About half of the bereaved families did not know how the patient evaluated their first SPC referral timing. However, patients at the end of life may find it difficult to report on their cancer experiences. In most cases, family responses are in line with patients' considerations and responses. Several studies conducted 3-12 months after patient death have found this to be an appropriate time frame for increasing participant inclusion and decreasing recall bias [13,35-38]. Second, some bereaved families may have under- or overreported SPC involvement, even though we used a detailed figure (Figure 1) to explain SPC. As families identified whether and when patients were referred to an SPC, the reported care involvement may have included medical practitioners other than those defined as SPC in this study. Additionally, about $5 \%$ of respondents indicated that patients had not been referred to SPC, and they may have misunderstood SPC as only including outpatient palliative care clinics or inpatient palliative care teams. SPC referral timing should be investigated separately from outpatient palliative care clinics, inpatient palliative care teams, and PCUs. Third, study participants were limited to family members of cancer patients who were admitted to and died in a PCU, where all could experience SPC. About $13 \%$ of all cancer patients who died in PCUs, family members who had serious psychological distress, and non-responders were not included in this analysis. Moreover, about half of all included patients were treated with anti-cancer therapy for more than one year, suggesting that the study sample might not be representative; therefore, the results might not apply to cancer patients who died in other settings or could not experience SPC. Finally, the questionnaire has not been fully validated although we developed it based on literature. However, there are currently no validated tools to investigate perceptions of appropriate SPC referral timing in cancer patients and their family members.

We showed that patients may evaluate early SPC involvement as appropriate based on their cancer experiences. However, it is not adequate to simply advocate early SPC involvement; future studies should identify methods for determining appropriate personalized SPC referral timing.

\section{Declarations}

\section{Funding}

This work was supported by the Ministry of Health, Labor, and Welfare in Japan (Health Labor Science Research Grant 20EA1009).

\section{Conflicts of interest}

This study was part of Japan Hospice and Palliative Evaluation Study 4, funded by the Japan Hospice Palliative Care Foundation and the corporation of Hospice Palliative Care Japan. The authors declare no conflicts of interest.

\section{Availability of data and material}

The data that support the findings of this study are available from the corresponding author, [K.T], upon reasonable request.

\section{Code availability}

Not applicable.

\section{Authors' contributions}

Keita Tagami: Conceptualization, Data curation, Formal analysis, Investigation, Methodology, Project administration, Writing - original draft, Writing - review and editing

Kento Masukawa: Conceptualization, Data curation, Investigation, Methodology, Project administration, Resources, Supervision, Writing - review and editing

Akira Inoue: Conceptualization, Investigation, Methodology, Writing - review and editing

Tatsuya Morita: Conceptualization, Investigation, Methodology, Writing - review and editing

Processing math: $100 \%$

Page 5/11 
Yusuke Hiratsuka: Investigation, Writing - review and editing

Mamiko Sato: Investigation, Writing - review and editing

Katsura Kohata: Investigation, Writing - review and editing

Noriaki Satake: Investigation, Writing - review and editing

Yoshiyuki Kizawa: Investigation, Supervision, Writing - review and editing

Satoru Tsuneto: Investigation, Supervision, Writing - review and editing

Yasuo Shima: Investigation, Supervision, Writing - review and editing

Mitsunori Miyashita: Conceptualization, Investigation, Methodology, Project administration, Resources, Supervision, Writing - review and editing

\section{Ethics approval}

Ethical approval for the study was granted by the institutional review boards of Tohoku University Hospital and all participating institutions (Approval No. 2018-2-290).

\section{Consent to participate}

The questionnaire was returned.

\section{Consent for publication}

Not applicable.

\section{Acknowledgements}

This work was supported by the Ministry of Health, Labor, and Welfare in Japan (Health Labor Science Research Grant 20EA1009). We would like to thank Editage (www.editage.com) for English language editing.

\section{References}

1. Hui D, Mori M, Watanabe SM et al (2016) Referral criteria for outpatient specialty palliative cancer care: An international consensus. Lancet Oncol 17:e552-e559

2. Hui D, Meng YC, Bruera S et al (2016) Referral criteria for outpatient palliative cancer care: A systematic review. Oncologist 21:895-901

3. Wong A, Vidal M, Prado B et al (2019) Patients' perspective of timeliness and usefulness of an outpatient supportive care referral at a comprehensive cancer center. J Pain Symptom Manage 58:275-281

4. Morita T, Fujimoto K, Namba M et al (2008) Palliative care needs of cancer outpatients receiving chemotherapy: An audit of a clinical screening project. Support Care Cancer 16:101-107

5. Glare PA, Semple D, Stabler SM et al (2011) Palliative care in the outpatient oncology setting: Evaluation of a practical set of referral criteria. J Oncol Pract 7:366-370

6. Wentlandt K, Krzyzanowska MK, Swami N et al (2012) Referral practices of oncologists to specialized palliative care. J Clin Oncol 30:4380-4386

7. Ferrell BR, Temel JS, Temin S et al (2017) Integration of palliative care into standard oncology care: American society of clinical oncology clinical practice guideline update. J Clin Oncol 35:96-112

8. Temel JS, Greer JA, Muzikansky A et al (2010) Early palliative care for patients with metastatic non-small-cell lung cancer. N Engl J Med 363:733-742

9. Zimmermann C, Swami N, Krzyzanowska M et al (2014) Early palliative care for patients with advanced cancer: A cluster-randomised controlled trial. Lancet 383:1721-1730

10. Bakitas MA, Tosteson TD, Li Z et al (2015) Early versus delayed initiation of concurrent palliative oncology care: Patient outcomes in the enable iii randomized controlled trial. J Clin Oncol 33:1438-1445

11. Temel JS, Greer JA, El-Jawahri A et al (2017) Effects of early integrated palliative care in patients with lung and GI cancer: A randomized clinical trial. J Clin Oncol 35:834-841

12. Temel JS, Jacobsen PB (2020) Palliative care in oncology: Continuing to build the evidence base and disseminate effective care models. J Clin Oncol 38:849-851

13. Masukawa K, Aoyama M, Morita T et al (2018) The japan hospice and palliative evaluation study 4: A cross-sectional questionnaire survey. BMC Palliat Care 17:66

14. Sakashita A, Kizawa Y, Kato M et al (2018) Development of a standard for hospital-based palliative care consultation teams in japan using a modified delphi method. J Pain Symptom Manage 56:746-751.e745

15. Sakashita A, Shutoh M, Sekine R et al (2019) Development of a consensus syllabus of palliative medicine for physicians in japan using a modified delphi Processing math: $100 \%$ 'Palliat Care 25:30-40 
16. Hui D, Kim SH, Roquemore J et al (2014) Impact of timing and setting of palliative care referral on quality of end-of-life care in cancer patients. Cancer 120:1743-1749

17. Morita T, Akechi T, Ikenaga M et al (2005) Late referrals to specialized palliative care service in japan. J Clin Oncol 23:2637-2644

18. Morita T, Miyashita M, Tsuneto S et al (2009) Late referrals to palliative care units in japan: Nationwide follow-up survey and effects of palliative care team involvement after the cancer control act. J Pain Symptom Manage 38:191-196

19. Costantini M, Toscani F, Gallucci M et al (1999) Terminal cancer patients and timing of referral to palliative care: A multicenter prospective cohort study. Italian cooperative research group on palliative medicine. J Pain Symptom Manage 18:243-252

20. Péus D, Newcomb N, Hofer S (2013) Appraisal of the Karnofsky performance status and proposal of a simple algorithmic system for its evaluation. BMC Med Inform Decis Mak 13:72--72

21. Miyashita M, Sanjo M, Morita T et al (2007) Good death in cancer care: A nationwide quantitative study. Ann Oncol 18:1090-1097

22. Hirai K, Miyashita M, Morita T et al (2006) Good death in japanese cancer care: A qualitative study. J Pain Symptom Manage 31:140-147

23. Miyashita M, Morita T, Sato K et al (2008) Good death inventory: A measure for evaluating good death from the bereaved family member's perspective. J Pain Smptom Manage 35:486-498

24. Kaasa S, Loge JH, Aapro M et al (2018) Integration of oncology and palliative care: A lancet oncology commission. Lancet Oncol 19:e588-e653

25. Hoerger M, Greer JA, Jackson VA et al (2018) Defining the elements of early palliative care that are associated with patient-reported outcomes and the delivery of end-of-life care. J Clin Oncol 36:1096-1102

26. Bakitas MA, El-Jawahri A, Farquhar M et al (2017) The team approach to improving oncology outcomes by incorporating palliative care in practice. $J$ Oncol Pract 13:557-566

27. Dalal S, Bruera S, Hui D et al (2016) Use of palliative care services in a tertiary cancer center. Oncologist 21:110-118

28. Yamamoto R, Kizawa Y, Nakazawa Y et al (2015) Outcome evaluation of the palliative care emphasis program on symptom management and assessment for continuous medical education: Nationwide physician education project for primary palliative care in japan. J Palliat Med 18:45-49

29. Yamamoto R, Kizawa Y, Nakazawa Y et al (2013) The palliative care knowledge questionnaire for peace: Reliability and validity of an instrument to measure palliative care knowledge among physicians. J Palliat Med 16:1423-1428

30. Nakazawa Y, Yamamoto R, Kato M et al (2018) Improved knowledge of and difficulties in palliative care among physicians during 2008 and 2015 in Japan: Association with a nationwide palliative care education program. Cancer 124:626-635

31. Sasahara T, Watakabe A, Aruga E et al (2014) Assessment of reasons for referral and activities of hospital palliative care teams using a standard format: A multicenter 1000 case description. J Pain Manage 47:579-587.e576

32. Fujisawa D, Umemura S, Okizaki A et al (2020) Nurse-led, screening-triggered, early specialised palliative care intervention programme for patients with advanced lung cancer: Study protocol for a multicentre randomised controlled trial. BMJ Open 10:e037759

33. Watanabe SM, Faily V, Mawani A et al (2020) Frequency, timing, and predictors of palliative care consultation in patients with advanced cancer at a tertiary cancer center: Secondary analysis of routinely collected health data. Oncologist 25:722-728

34. Temel JS, Sloan J, Zemla T et al (2020) Multisite, randomized trial of early integrated palliative and oncology care in patients with advanced lung and gastrointestinal cancer: Alliance a221303. J Palliat Med 23:922-929

35. Costantini M, Beccaro M, Merlo F (2005) The last three months of life of italian cancer patients. Methods, sample characteristics and response rate of the italian survey of the dying of cancer. Palliat Med 19:628-638

36. Breen LJ, Aoun SM, O'Connor M (2015) The effect of caregiving on bereavement outcome: Study protocol for a longitudinal, prospective study. BMC Palliat Care 14:6

37. Maciejewski PK, Zhang B, Block SD et al (2007) An empirical examination of the stage theory of grief. JAMA 297:716-723

38. Aoyama M, Morita T, Kizawa Y et al (2017) The Japan hospice and palliative care evaluation study 3: Study design, characteristics of participants and participating institutions, and response rates. Am J Hosp Palliat Med 34:654-664

\section{Tables}

Table 1: Background information and clinical characteristics of the patients and their bereaved family members 
Patients $(n=515)$

Age (years), Mean (S.D. ${ }^{a}$ )

$76.0(11.3)$

Sex

Male

$284(55.1)$

Female

$231(44.9)$

Primary tumor sites

\begin{tabular}{ll}
\hline Lung & $125(24.3)$ \\
\hline Esophagus, Stomach & $68(13.2)$ \\
\hline Colon, Rectum & $64(12.4)$ \\
\hline Pancreas & $58(11.3)$ \\
\hline Liver, Hepatobiliary system & $49(9.5)$ \\
\hline Urinary tract & $30(5.8)$ \\
\hline Breast & $25(4.9)$ \\
\hline Gynecological & $25(4.9)$ \\
\hline Others & $71(13.7)$
\end{tabular}

Duration of anti-cancer treatment

\begin{tabular}{ll} 
More than 3 years & $134(26.0)$ \\
\hline 1 to 3 years & $163(31.7)$ \\
\hline 6 months to 1 year & $76(14.8)$ \\
\hline 3 to 6 months & $52(10.1)$ \\
\hline Less than 3 months & $79(15.3)$ \\
Unknown & $11(2.1)$
\end{tabular}

Number of days Palliative care unit stays ${ }^{b}$

Days, Median (IQR $\left.{ }^{\mathrm{C}}\right) \quad 20(10-41)$

Bereaved family members $(n=515)$

Age (years), Mean (S.D. ${ }^{a}$ )

$62.9(11.6)$

Sex

$\begin{array}{ll}\text { Male } & 165(32.4) \\ \text { Female } & 340(66.8) \\ \text { Missing } & 10(1.9)\end{array}$




\begin{tabular}{ll} 
Child & $212(41.7)$ \\
\hline Spouse & $199(39.2)$ \\
\hline Sibling & $33(6.4)$ \\
\hline In-law & $28(5.4)$ \\
\hline Parent & $16(3.1)$ \\
\hline Other & $17(3.3)$ \\
\hline Missing & $10(1.9)$ \\
\hline & \\
\hline Interval from patient death to study & $323.5(265-409)$ \\
\hline Days, Median (IQR) & $79.3(14.4)$ \\
\hline & \\
\hline Score of GDI ${ }^{d}$ Mean (S.D.) & \\
\hline${ }^{a}$ Standard deviation & \\
\hline${ }^{b}$ The periods of staying at palliative care unit from last admission to patient death \\
\hline${ }^{\text {Interquartile range }}$
\end{tabular}

Table 2. Bereaved families-perceived appropriate referral timing to specialized palliative care service

\begin{tabular}{ll} 
Timing & Agree; $\mathrm{n}(\%)$ \\
\hline Cancer patients have no physical and/or psychological distress & \\
\hline At diagnosis of advanced or incurable cancer & $363(75.5)$ \\
\hline During anti-cancer treatment & $338(71.9)$ \\
\hline No further standard anti-cancer treatment options but independent ADL ${ }^{* 1}$ & $427(89.5)$ \\
\hline No further standard anti-cancer treatment options and dependent ADL ${ }^{* 2}$ & $440(93.6)$
\end{tabular}

${ }^{*}$ Activities of Daily Living

${ }^{\star 2}$ Functional stats as Karnofsky Performance Status Scale being forty or less

\section{Figures}



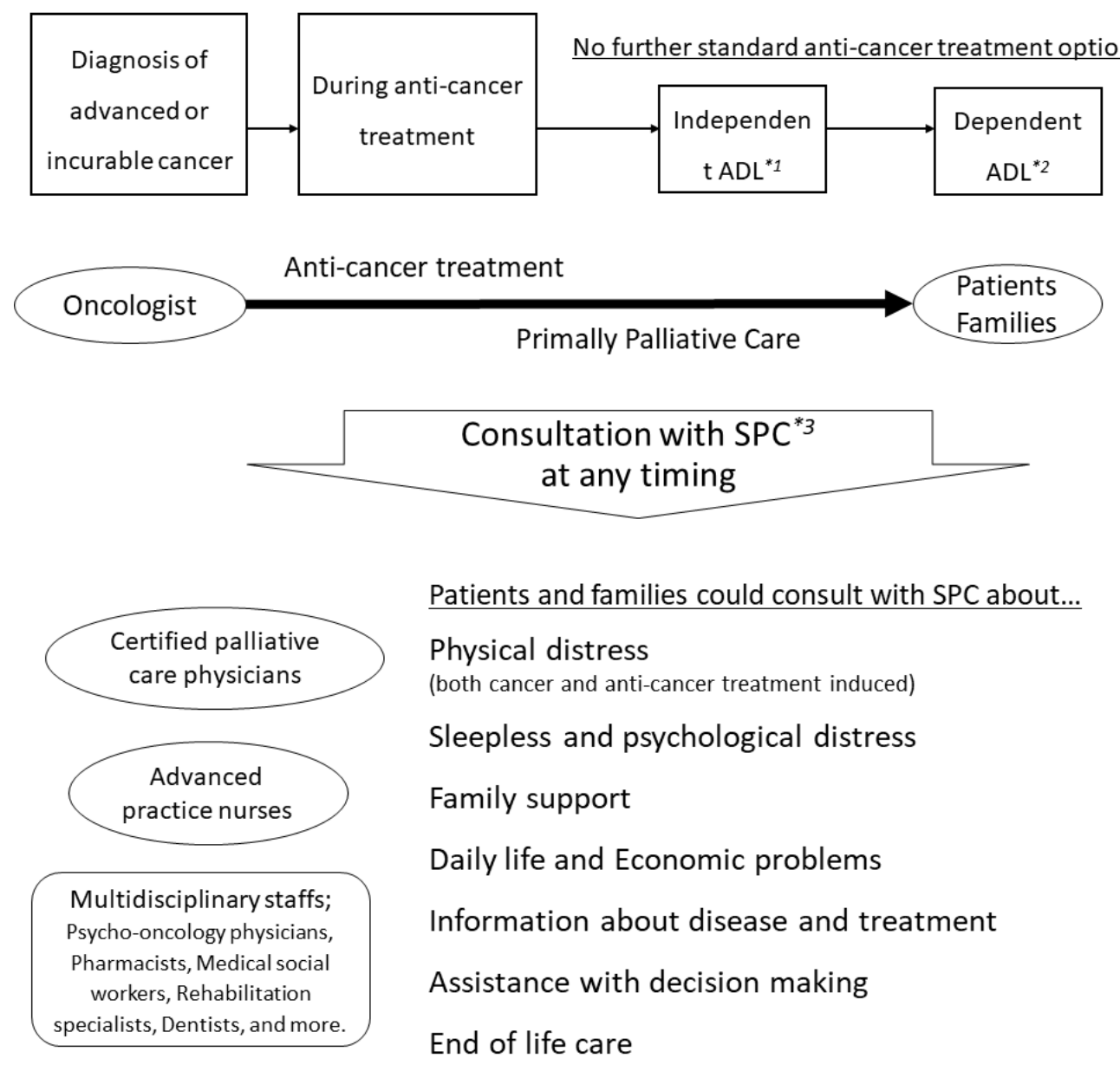

\section{Figure 1}

Explanation of SPC*3 in questionnaire Specialized palliative care service was defined as an outpatient palliative care clinic or inpatient palliative care team comprising certified palliative care physicians, advanced-practice nurses, and multidisciplinary palliative care staff (psycho-oncology physicians, pharmacists, medical social workers, rehabilitation specialists, dentists, etc.) who support the patients. *1Activities of Daily Living *2Functional stats as Karnofsky Performance Status Scale $\leq 40$ *3Specilized Palliative Care service 


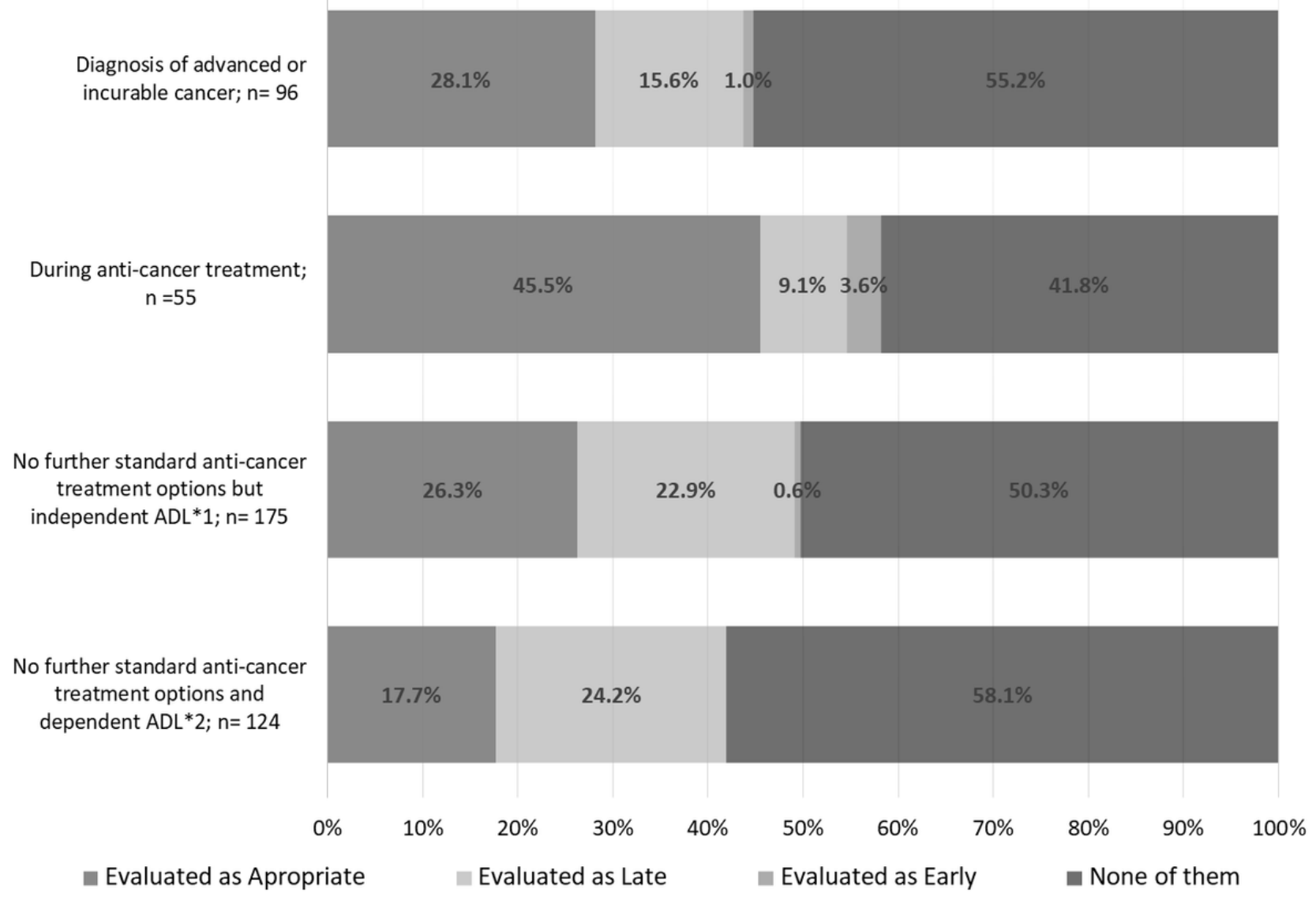

Figure 2

\section{Figure 2}

Patients' evaluation of their first SPC referral timing according to the actual time physicians first referred patients to SPC. The evaluation was classified into four referral timing groups: "late" and "too late" were coded as "evaluated as late," and "early" and "too early" were coded as "evaluated as early," "appropriate" was coded as "evaluated as appropriate" and "none of them" (bereaved families did not know how patients had evaluated their first SPC referral timing).

*1Activities of Daily Living *2Functional stats as Karnofsky Performance Status Scale $\leq 40$ 